\title{
COERCIVITY OF ULTRATHIN MAGNETIC FILMS WITH PERPENDICULAR ANISOTROPY
}

\author{
V. ZablotskiI ${ }^{a}$, M. Kisielewshi ${ }^{b}$, O. Tsiganenko ${ }^{c}$ AND J. Ferre ${ }^{d}$ \\ a Physics Department, Donetsk State University, 340055 Donetsk, Ukraine \\ ${ }^{b}$ Institute of Physics, University at Białystok, Lipowa 41, 15-424 Białystok, Poland \\ 'Institute of Physics, Academy of Sciences of Czech Republic \\ Na Slovance 2, 18040 Praha 8, Czech Republic \\ ${ }^{d}$ Lab. de Phys. des Solides, URA CNRS 02, Université Paris-Sud, 91405 Orsay, France
}

\begin{abstract}
The calculation of the temperature dependence of the domain wall coercivity of ultrathin magnetic films with perpendicular anisotropy is reported. In this case, the magnetization reversal is supposed to occur through domain wall motion. The proposed model takes into account thermal bending fluctuations of the domain wall, which is pinned by crystal defects such as grain boundaries. A fitting procedure, applied to recently published experimental data on the field induced domain wall velocity in Au/Co films, allows us to find out realistic values of the involved parameters.
\end{abstract}

PACS numbers: 75.60.-d, 75.60.Ch, 75.70.Ak

In many experiments, which had been performed for ultrathin ferromagnetic films and multilayers, either a nonlinear or almost linear law of the coercivity decrease with the increase in the film temperature has been found (for a review see Refs. [1-4]). This decrease can be understood as coming from a mechanism of thermally activated domain wall (DW) motion. Indeed, the DW coercivity at $T=0$ is determined by a value of the energy barrier which separates two different positions of a DW segment. At $T>0$ the energy of thermal oscillations of the DW segment favours to overcome the coercivity barrier and decreases its effective value.

The goal of this paper is to thermodynamically examine the bending oscillation of DW and the mechanism of their movement in ultrathin magnetic films with a perpendicular anisotropy. The basis is the DW vibration model similar to that applied in Ref. [5] for the description of the bend fluctuations of long molecules.

Let us suppose that a segment of the DW is pinned to two defects (or to grain boundaries) and that the distance between them is $2 R$. We consider a limp type of DW and a weak DW pinning regime. For $T>0$ the DW can change its form owing to the effect of the temperature fluctuations. We will describe the form of the DW bend by the following equation $y=b / c h(x / R)$ (here $b$ is the amplitude of the DW bending). The magnetic field is directed perpendicular to the film surface. 
The probability of standing of the DW in a bent state is given by the usual thermodynamic expression: $W=C \exp (-\Delta F / k T)$, where $\Delta F$ is the change of the free energy of the bent DW in respect to a not bent one, $k$ is the Boltzmann constant, $T$ is the film temperature, $C$ is a constant. We can write the change of the free energy of DW as

$$
\Delta F=-2 M_{\mathrm{S}} H \Delta S h+\gamma h \Delta L,
$$

where $M_{\mathrm{S}}$ is the saturation magnetization of the film, $H$ is the magnetic field strength, $\Delta S$ and $\Delta L$ is the change of the area under the DW and the length of the boundary, respectively, $h$ is the film thickness and $\gamma$ is the DW surface energy density. In a more general case expression (1) additionally has to contain the energy of interaction between the DW and other pinning centres as well as the change of the magnetostatic energy. But the first of them is negligible when the average period of defects exceeds the DW width (the DW width is the action radius of a pinning potential [6]). This condition is usually fulfilled for films with a polycrystalline structure. The magnetostatic energy in ultrathin films with large domains only renormalizes the anisotropy energy [7] and therefore it can be also omitted. Calculations give the following formulas for $\Delta S$ and $\Delta L$ :

$$
\Delta S=2 b R \arctan \left[\left(e^{2}-1\right) / 2 e\right], \quad \Delta L=\left(b^{2} \tanh ^{3} 1\right) / 3 R,
$$

where $e=2.71828 \ldots$. Now we can calculate the average amplitude of the DW bending

$$
\langle b\rangle=C \int_{-\infty}^{\infty} b \exp \left(-\frac{\Delta F}{k T}\right) \mathrm{d} b,
$$

where $C$ is found from the normalization condition.

The calculation of the average $b$ parameter is fulfilled in accordance with $(1-3)$. It gives

$$
\langle b\rangle=\frac{6 M_{\mathrm{S}} H R^{2}}{\gamma \tanh ^{3} 1} \arctan \left(\frac{e^{2}-1}{2 e}\right) .
$$

In the frame of our model the DW is oscillating close to the equilibrium position. It is determined by the expression $b_{0} \approx M_{\mathrm{S}} H R^{2} / \gamma$, which follows from the balance of the pressures resulting from the magnetic field and the DW curvature. The dispersion of the DW displacement is calculated as

$$
\sigma_{b}=\sqrt{\left\langle b^{2}\right\rangle-\langle b\rangle^{2}}=\sqrt{\frac{3 k T R}{2 \gamma h \tanh ^{3} 1}} .
$$

So the DW is oscillating near the equilibrium positions (expression (4)) with the dispersion defined by (5).

Let us consider a DW thermoactivation jump from one defect to another one. When $H$ increases, the DW bends to the side of the domain with $M$ antiparallel to $\boldsymbol{H}$ and the bend amplitude rises proportionally to $H$ (see expression (4)). At the same time the dispersion (5) does not depend on $H$. It is defined only by the film temperature. The DW could be found with the almost unit probability in the following range of displacement: $\langle b\rangle-3 \sigma \leq b \leq\langle b\rangle+3 \sigma$.

Let $d_{0}$ be a characteristic defect period or a typical grain size. In the latter case we can assume that the DW is pinned to islands or grain boundaries [8]. 
If $\langle b(H)\rangle=d_{0}$ is reached while increasing the field, the DW goes over to the next defect. But it is not a thermally activated DW motion. The mechanism of the thermally activated DW motion consists of the removal of the DW to the next defect owing to thermofluctuations at $\langle b(H)\rangle\left\langle d_{0}\right.$. Let us assume that the thermally activated movement leads up the depinning of DW if the following condition is fulfilled:

$$
d_{0}=\langle b\rangle+3 \sigma_{b} .
$$

Thus, DW with the bend amplitude $b(H, T) \geq 3 \sigma_{b}+\langle b(H, T)\rangle$ can be considered as unpinned DW. A similar condition of a thermally activated wall release had been used before in Ref. [9]. Another condition of the DW depinning was used in Ref. [10]. Taking (6) into account we can calculate the activation volume, the activation energy, and the coercive field as a function of the film temperature. Indeed, as it follows from (2) and (6) the activation volume (Barkhausen volume) is equal to

$$
V_{a}=h\left[S\left(\langle b\rangle+3 \sigma_{b}\right)-S(\langle b\rangle)\right]=6 R h \sigma_{b} \arctan \left(\frac{e^{2}-1}{2 e}\right) .
$$

In (7) $\sigma_{b}$ is defined by the expression (5). The activation volume grows with increasing the film temperature. It is in qualitative agreement with experimental data [11].

Let us find the coercive field of DW in the case when in a film DWs are pinned to the grain boundaries, i.e. $d_{0}=2 R$. We will account that the DW goes over to the next defect if the condition (6) is fulfilled. We can find the coercive field from (6), (4), and (5) in the following form:

$$
H_{\mathrm{c}}=H_{\mathrm{c}}(0)\left(1-3 \sqrt{\frac{3 k T}{4 \gamma h \tanh ^{3} 1 d_{0}}}\right),
$$

where $H_{\mathrm{C}}(0)=2 C_{1} \gamma / 3 M_{\mathrm{s}} d_{0}, C_{1}=\tanh ^{3} 1 / \arctan \left(\frac{e^{2}-1}{2 e}\right) \approx 0.51$.

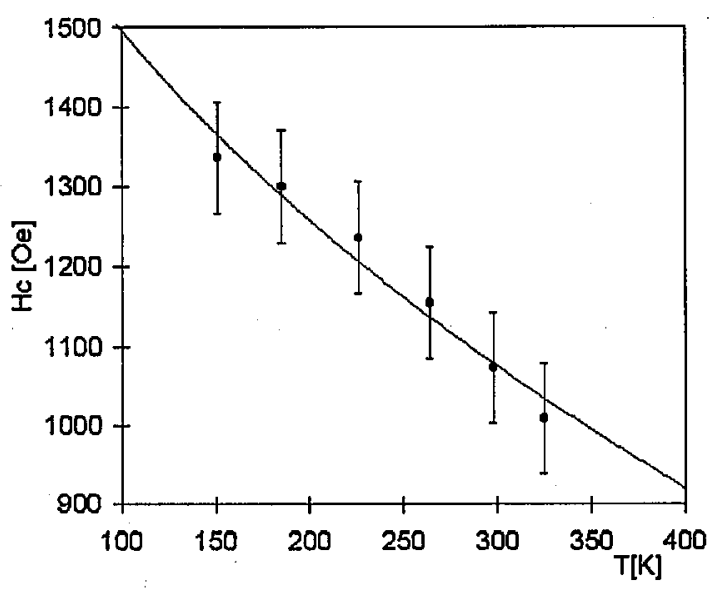

Fig. 1. Experimental [12] and fitted (8) dependences of $H_{\mathrm{c}}$ on temperature. 
After performing a fitting procedure using formula (8) and experimental $H_{\mathrm{c}}(T)$ dependence taken from Ref. [12], the following values of film parameters could be found: $d_{0} \cong 20.0 \mathrm{~nm}, \gamma=17.1 \mathrm{erg} / \mathrm{cm}^{2}$. Calculations were performed for $h_{\mathrm{Co}}=0.8 \mathrm{~nm}, M_{\mathrm{S}}=1400 \mathrm{emu} / \mathrm{cm}^{3}$ and assuming no temperature dependence of $M_{\mathrm{S}}$ and $\gamma$. The comparison of the experimental $H_{\mathrm{c}}(T)$ dependence and the fitted curve is shown in Fig. 1.

It should be noted that the calculated $\gamma$ parameter is in good agreement with the one given in Ref. [13] for Co/Pd films. And $d_{0}$ is close to the typical crystallite size (about $10 \mathrm{~nm}$ ) of Co/Au films [11]. The Barkhausen volume estimated from (5) and (7) for film parameters given above is $V_{a}=10^{-18} \mathrm{~cm}^{3}$ at room temperature and it agrees with experimental data for Co/Au films reported in Ref. [11].

So, we can conclude that in ultrathin films the DW motion is well described by the thermodynamic approach in which the bend fluctuations of the DW shape are taken into account. Experiment of visualisation of DW propagation in ultrathin Co/Pt films performed recently in Ref. [14] directly shows that the DW propagates by bending through small jumps from one pinning centre to another.

This work was partially supported by ESF NANOMAG Program and Polish grant No. 2P03B06515.

\section{References}

[1] Y.D. Yao, Y. Liou, J.C.A. Huang, J. Appl. Phys. 79, 6533 (1996).

[2] T. Thomson, K. O'Grady, IEEE Trans. Magn. 33, 795 (1997).

[3] J.P. Jamet, P. Meyer, V. Grolier, J. Magn. Soc. Jpn. 20, Suppl. NS1, 217 (1996).

[4] A. Berger, H. Hopster, Phys. Rev. Lett. 73, 193 (1994).

[5] L.D. Landay, E.M. Liphsitz, Statistical Physics, Part I, Moscow 1964, p. 565.

[6] H.R. Hilzinger, H. Kronmuller, J. Magn. Magn. Mater. 2, 11 (1976).

[7] J. Ferre, V. Grolier, P. Meyer, Phys. Rev. B 55, 15092 (1997).

[8] M. Fahnle, B. Barbara, H. Kronmuller, Phys. Status Solidi B 96, 343 (1980).

[9] K.B. Klaassen, J.C.L. Van Peppen, IEEE Trans. Magn. 30, 375 (1994).

[10] W. Schmidt, J. Magn. Magn. Mater. 114, L1 (1992).

[11] A. Kirilyuk, J. Ferre, D. Renard, IEEE Trans, Magn. 29, 2518 (1993).

[12] A. Kirilyuk, J. Ferre, V. Grolier, J.P. Jamet, D. Renard, J. Magn. Magn. Mater. 171, 45 (1997).

[13] J.R. Barnes, S.J. O'Shea, J. Appl. Phys. 76, 2974 (1994).

[14] S. Lemerle, J. Ferre, A. Thiaville, in: Magnetic Hysteresis in Novel Magnetic Material, Ed. G.C. Hadjipanayis, Kluwer Academic Publ., Dordrecht 1997, p. 537. 